\title{
Faraday rotation retrieval using SMOS radiometric data
}

\author{
Ignasi Corbella, Lin Wu, Student Member, IEEE, \\ Francesc Torres, Senior Member, IEEE, Nuria Duffo, Member, IEEE, and \\ Manuel Martín-Neira, Senior Member, IEEE
}

\begin{abstract}
Faraday rotation is dynamically computed from L-Band radiometric data acquired by the satellite SMOS (Soil Moisture and Ocean Salinity). Improved full polarimetric image reconstruction and smart spatio-temporal filtering are used to obtain good estimations of Faraday rotation, which compare very well with the values theoretically predicted from available ionosphere and geomagnetic field data.
\end{abstract}

\section{Index Terms}

Faraday rotation, L-band radiometry, Interferometric synthetic aperture radiometry, SMOS

\section{INTRODUCTION}

SMOS is an European Space Agency (ESA) mission designed to provide global maps of soil moisture over land and sea surface salinity over oceans [1]. It has a single payload named MIRAS (Microwave Imaging Radiometer with Aperture Synthesis) [2] responsible of measuring the polarimetric brightness temperature of the Earth surface, used in scientific models for estimating

This work was supported by the European Space Agency and Deimos Enginheria (Portugal) under contract DME-AS-2010-001; and by the Spanish Ministry of Science and Technology (MCYT) and FEDER projects TEC2011-25865 and AYA2012-39356C05-01

L. Wu, I. Corbella, F. Torres, and N. Duffo are with the Remote Sensing Laboratory, Universitat Politècnica de Catalunya, Barcelona, Spain (e-mail: corbella@tsc.upc.edu).

M. Martín-Neira is with the European Space Agency (ESA-ESTEC) Noordwijk, The Netherlands (e-mail: Manuel.MartinNeira@esa.int) 
both geophysical parameters as well as other products, such as for example ice cover or winds over oceans.

In radiometric measurements from space, as microwave radiation from earth propagates through the ionosphere, the electromagnetic field components are rotated an angle, called Faraday rotation, that depends on the total ionospheric electron content (TEC), the frequency and the geomagnetic field. At the SMOS operating frequency $(1.4135 \mathrm{GHz})$, Faraday rotation is not negligible and must be corrected. It is presently estimated using a classical formulation [3] that makes use of data provided by external sources to get the necessary TEC and geomagnetic field.

Improved data processing requires getting the Faraday rotation directly from radiometric data in a continuous way. This is possible using the full polarimetric capability of the SMOS sensor [4], which now can be fully exploited due to recent improvements in the image reconstruction algorithms, especially regarding the third and fourth stokes parameters [5]. However, estimating Faraday rotation out of current SMOS radiometric data is not as straightforward as it may seem. MIRAS has relatively poor radiometric sensitivity (thermal noise) and accuracy (spatial bias), and this makes it difficult to do it with the required accuracy.

\section{FARADAY ROTATION}

The angle of rotation in the polarization of a linearly polarized electromagnetic field propagating through the ionosphere can be approximately expressed (in degrees) by the following equation [3] [6]:

$$
\Omega_{f}=1.355 \times 10^{4} f^{-2} B_{0} \cos \Theta_{B} \sec \theta \mathrm{VTEC}
$$

with $f$ the frequency in $\mathrm{GHz}$ (1.4135 for SMOS), $B_{0}$ the geomagnetic field in Tesla, $\Theta_{B}$ the angle between the magnetic field and the wave propagation direction, $\theta$ the angle between the wave propagation direction and the vertical to the surface and VTEC the vertical total electron content in TEC units $\left(10^{16}\right.$ electrons $\left./ \mathrm{m}^{2}\right)$.

In SMOS processing, the geomagnetic field is obtained from the database of the International Geomagnetic Reference Field (IGRF) [7]. The ionospheric electron content VTEC is read from a SMOS auxiliary data file, the so-called "consolidated TEC", which gives the total electron content of the ionosphere for all the globe at intervals of 2 hours. It is built periodically from IGSG files provided by the International GNSS Service (IGS) and it is available with a delay typically 
of 15 days since radiometric data acquisition. Both the geomagnetic field and the vertical total electron content are given at the Ionospheric Pierce Point (IPP) at a geodetic altitude of $400 \mathrm{~km}$. For a given spatial direction from satellite to earth, the longitude and latitude coordinates of the path crossing point through an altitude of $400 \mathrm{~km}$ is computed. Both the geomagnetic field and TEC maps are then interpolated at these coordinates and also a time interpolation of the TEC map is applied. Basic geometry is used to compute the two angles of (1) at this point.

\section{RETRIEVAL FROM RADIOMETRIC DATA}

Every 2.4 seconds the radiometer onboard SMOS provides a full polarimetric L-band brightness temperature image in a large field of view over the earth, with a swath of about $1000 \mathrm{~km} \mathrm{[2].}$ The polarization basis $(x, y)$ is defined by the identical orientation of all the 69 antennas of the instrument. At each spatial direction from instrument to earth surface, this polarization frame is rotated a different angle with respect to the nominal ground-defined "horizontal" and "vertical" polarizations $(h, v)$. Additionally, due to propagation of the electromagnetic wave through the ionosphere, the polarization axes rotate an angle equal to the Faraday rotation. So, the total angle of rotation at any spatial direction is the sum of the geometrical term $\varphi$, specific of the platform attitude and instrument orientation, and the Faraday rotation $\Omega_{f}$. Assuming that the horizontal and vertical polarizations of the ground emission are uncorrelated, the instrument full polarimetric measurement at each snapshot is related to the ground emission by the following rotation matrix [4]:

$$
\left[\begin{array}{c}
T_{B}^{x x} \\
2 T_{B}^{x y} \\
T_{B}^{y y}
\end{array}\right]=\left[\begin{array}{cc}
\cos ^{2}\left(\varphi+\Omega_{f}\right) & \sin ^{2}\left(\varphi+\Omega_{f}\right) \\
-\sin 2\left(\varphi+\Omega_{f}\right) & \sin 2\left(\varphi+\Omega_{f}\right) \\
\sin ^{2}\left(\varphi+\Omega_{f}\right) & \cos ^{2}\left(\varphi+\Omega_{f}\right)
\end{array}\right]\left[\begin{array}{c}
T_{B}^{h h} \\
T_{B}^{v v}
\end{array}\right]
$$

where all superscripts refer to polarization frames. By dividing the second row of the above system of equations by the sum of first and third, the Faraday rotation can be solved:

$$
\Omega_{f}=-\varphi-\frac{1}{2} \arctan \left(\frac{2 \Re e\left(T_{B}^{x y}\right)}{T_{B}^{x x}-T_{B}^{y y}}\right)
$$

This equation is equivalent to (22) of [6], although here it is written in terms of the SMOS level 1 products. In principle, it can be used to get an instantaneous value of Faraday rotation for all spatial directions in the large SMOS field of view, except those around $45^{\circ}$ of total rotation for which $T_{B}^{x x} \approx T_{B}^{y y}$. 
In practice, due to the relatively large thermal noise of SMOS images, high errors are introduced into the retrievals when equation (3) is used for a single snapshot. Averaging a number of snapshots reduces the thermal noise, but also washes out the natural variation of the Faraday rotation when a large number of them is used. Additionally, spatial errors of SMOS images due to calibration inaccuracy, image reconstruction artifacts and antenna pattern uncertainty [8] limit the quality of the retrievals. It is not possible, then, to use this procedure to directly get instantaneous values of Faraday rotation at each pixel of the SMOS field of view.

A way to overcome this problem is by computing (3) only in a reference area of the SMOS field of view where the quality of the data is higher. In [9] it is shown that the area around boresight has the lowest thermal noise and, at the same time, it is known to present the minimum image reconstruction artifacts [5], so it is the zone where better retrievals are expected. In particular, it is important to restrict the area to the alias-free field of view which, in addition, does not include the pixels with $45^{\circ}$ geometrical rotation. If Faraday rotation in a circle around boresight is averaged, both spatial bias and thermal noise contamination are significantly minimized and the quality of the retrievals increases.

As an example, figure 1 shows the theoretical Faraday rotation of a typical SMOS snapshot in the extended alias-free field of view. The spatial variation is due to the change in the orientation between the signal path and the geomagnetic field at each direction, and also to variations in the total electron content of the Ionosphere. It is quite smooth, so the spatial average in a circle around the origin is similar to the boresight value $(\xi=0, \eta=0)$. In this particular case, the average in a circle of radius $r=0.3$ (shown in the figure) is $5.23^{\circ}$, while the value at boresight is $5.1^{\circ}$. The plot at the right shows both values as a function of the boresight latitude for several snapshots over the Pacific ocean. The statistical difference between the Faraday rotation at the boresight and that averaged within $r<0.3$, computed for all the orbits shown in figure 5 (right), is $0.0215^{\circ}$ in mean and $0.124^{\circ}$ in standard deviation.

\section{RESUlTS USING SMOS DATA}

Several orbits of SMOS data have been processed up to level 1B using the Miras Testing Software (MTS), a tool developed by the authors and currently used as breadboard in the development of new versions of the SMOS level 1 operational processor (L1OP). At the moment of writing this document, MTS is cross-validated with version 620 of L1OP, released in early 

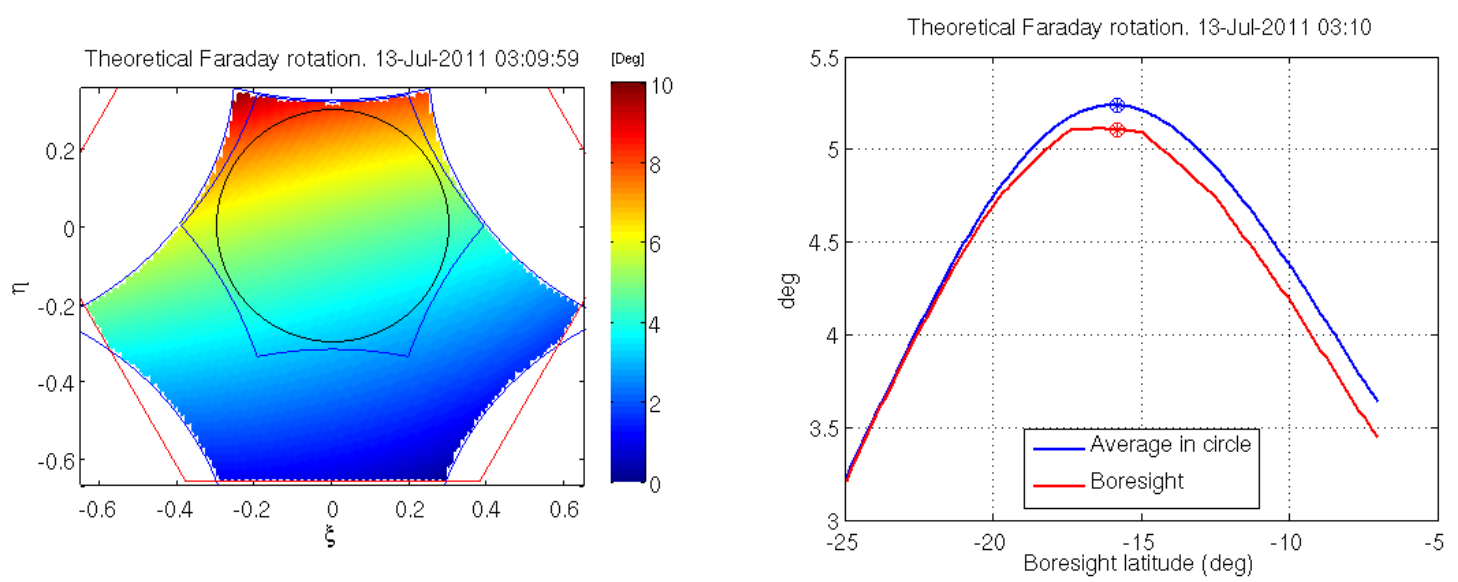

Fig. 1. Left: Theoretical Faraday rotation in the SMOS extended alias-free field of view. The coordinates $\xi$ and $\eta$ are the director cosines defined with respect to the radiometer reference frame. The alias-free field of view and a circle of $r=0.3$ are also shown in the figure. Right: Theoretical Faraday rotation as a function of latitude at boresight (instrument pointing) showing both the average in the circle $r=0.3$ and the boresight value.

2014.

Figure 2 shows the Faraday rotation retrieved from SMOS radiometric data for a descending orbit over the Pacific ocean in July 2011. Each grey dot is the average of (3) in a circle of radius $r=0.3$ (about 225 reconstructed beams) for a single snapshot. Clearly, even after averaging all pixels in the reference area, there is some scatter from snapshot to snapshot, mainly due to thermal noise. The somewhat larger dispersion seen at high northern latitudes might be due to the presence of Radio Frequency Interference (RFI). It has been mitigated to some extent by keeping in (3) only brightness temperatures lower than $330 \mathrm{~K}$. A running average in time provides the necessary filtering in order to reduce the noise fluctuations while keeping the variation of the Faraday rotation along the orbit. A triangular filter of length 41 (about 100 seconds) has been used to produce the black line shown in figure 2. It clearly reduces the dispersion while keeping the natural variations of the Faraday rotation. The plot also shows in red the expected value (1) which compares very well to the filtered retrieval, particularly regarding the location of the maximum and the general shape. To be more precise, the scatter plot and histogram of figure 3 represent the difference between both red and black lines of figure 2. For this particular orbit the standard deviation of the difference is about $\sigma \approx 0.95^{\circ}$ with zero mean.

The main transitions between ocean and land are marked by vertical lines in cyan. They are 

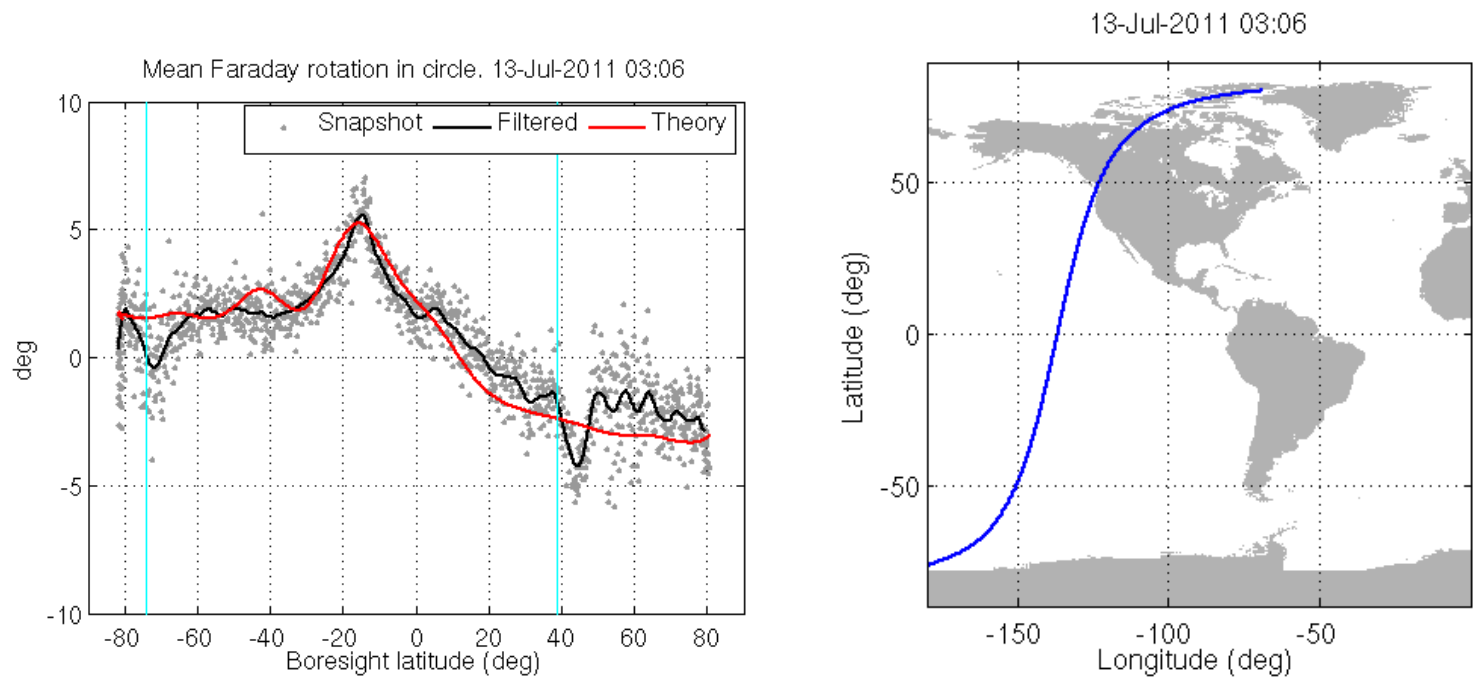

Fig. 2. Faraday rotation in the descending orbit of July 2011 shown at right. Grey dots are the individual retrievals at each shapshot, black line is the running average of the same data and red line are theoretical predictions. The cyan lines mark the latitudes where the circle of $\mathrm{r}=0.3$ enters land.
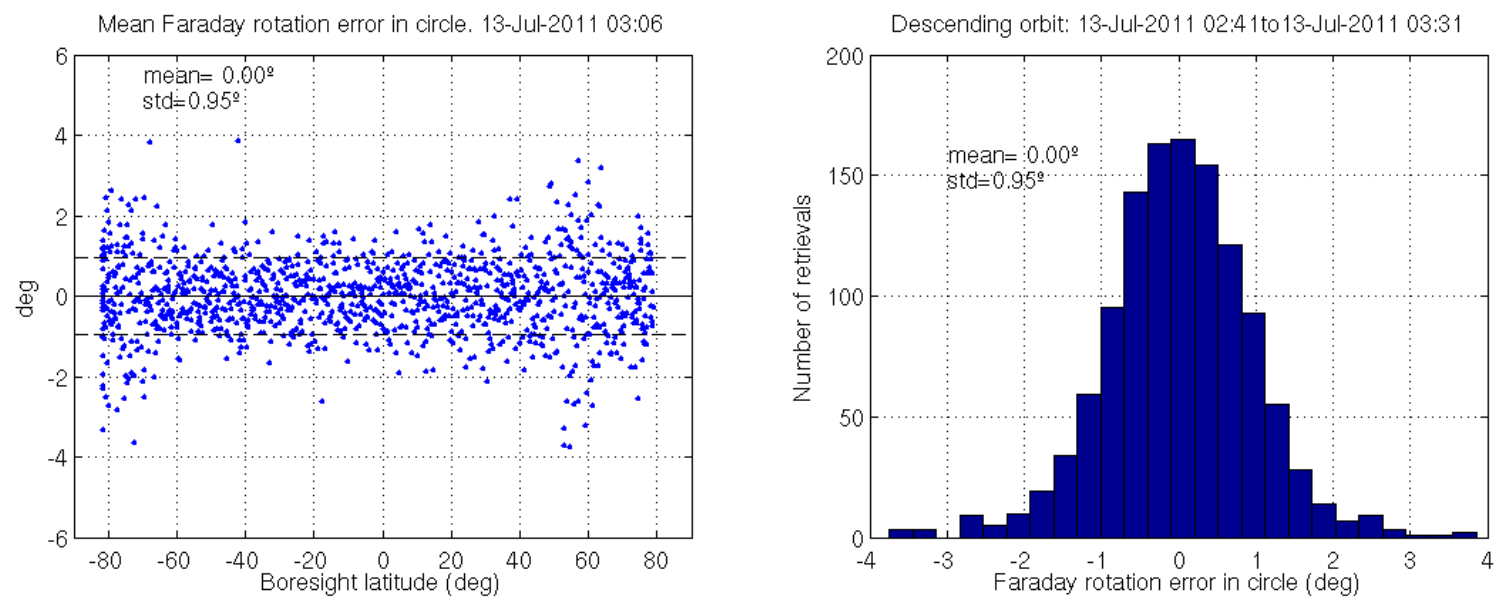

Fig. 3. Difference between faraday rotation retrieved from radiometric data (black line of figure 2) and theoretical predictions (red line of figure 2). Left: scatter plot. Right: histogram

drawn at those latitudes in which the circle of $r=0.3$ just enters land regions. Here the retrieved Faraday rotation shows large dips attributed hypothetically to the limited spatial resolution of the instrument. As pointed out in [10], all pixels including both land and sea induce spikes in the third stokes parameter due to the asymmetry of the scene within them. In the case of averaging all the retrievals in a relatively large area of the field of view, as it is the case here, this effect is 
mitigated since it only affects few pixels in the average. Nevertheless, it is perfectly noticeable in figure 2. In high southern latitudes the dip does not match with the land transition due to the presence of sea ice.

Results covering a larger time span are provided in figure 4. In this case, two sets of about 8 hours of continuous measurements separated one year (May 2011 and May 2012) have been considered. Each set includes a handful of ascending and descending orbits over land, sea and ice and the corresponding transitions. For reference, the orbits are depicted in figure 5. Taking into account that the motion of the satellite is from right to left, there are four ascending and five descending orbits in the data of May 2011; and five ascending and four descending in that of May 2012. The SMOS orbit has ascending node at 6:00 A.M, so all ascending orbits correspond to the morning local time while the descending ones are at afternoon. This has an important impact in the Faraday rotation measurements.

The plots of figure 4 show in red the theoretical Faraday rotation (1) and in black the retrieval from radiometric data using (3) with the procedure described before. In this case, a triangular filter of 101 samples has been used to further reduce the noise. In all cases the plots are the average of Faraday rotation within the circle of radius $r=0.3$. Clearly the instantaneous retrieval using radiometric data is able to track dynamic changes in the rotation angle. In general a fairly good agreement between both red and black traces is observed, with an average difference of $0.3^{\circ}$ for data of 2011 and $0.2^{\circ}$ for 2012 and standard deviations of $1.44^{\circ}$ and $1.38^{\circ}$ respectively.

The Faraday rotation peaks in descending orbits (labeled " $D$ " in the plot) are well located in all cases, although their value has different levels of matching depending on the orbit considered, which in the end depends on longitude (see the orbit plots in figure 5). Differences between theory and measurement are attributed to approximations in (1), residual radiometric errors in the polarimetric brightness temperature and possible time and space variations of Geomagnetic field and TEC at a local scale.

Ripples due to land/ice-sea transitions are clearly noticed in the plots, especially in areas with large variability, such as the Mediterranean Sea and Europe in ascending orbits. Probably this is one of the reasons of the poorer matching observed. Also RFI presence, although not spoiling completely the results, makes important differences in the some areas. For example in ascending orbits passing through middle East, as marked with an arrow in the orbits and results of 2012. 

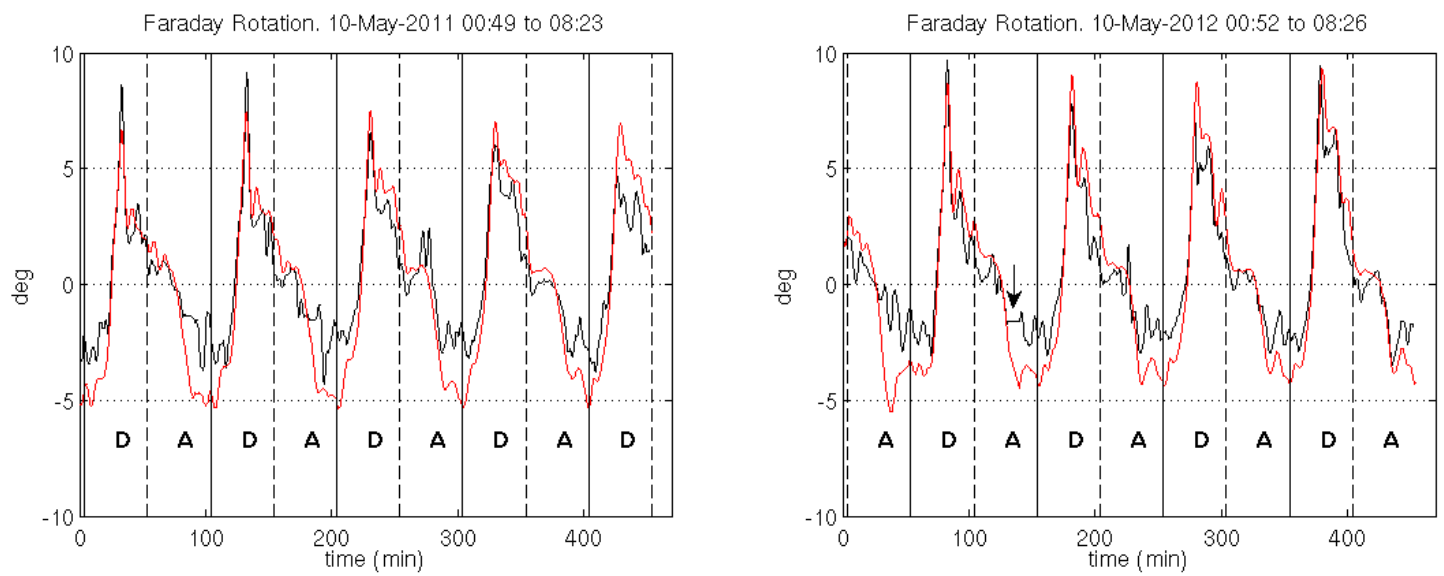

Fig. 4. Retrieved Faraday rotation from SMOS data in 10-May-2011 (left) and 10-May-2012 (right). Black curves are retrieved from radiometric data in the circle of radius 0.3 using a running average of 101 samples with triangular window. Red curves are the theoretical predictions using (1). Letters "A" and "D" stand for ascending and descending orbits respectively
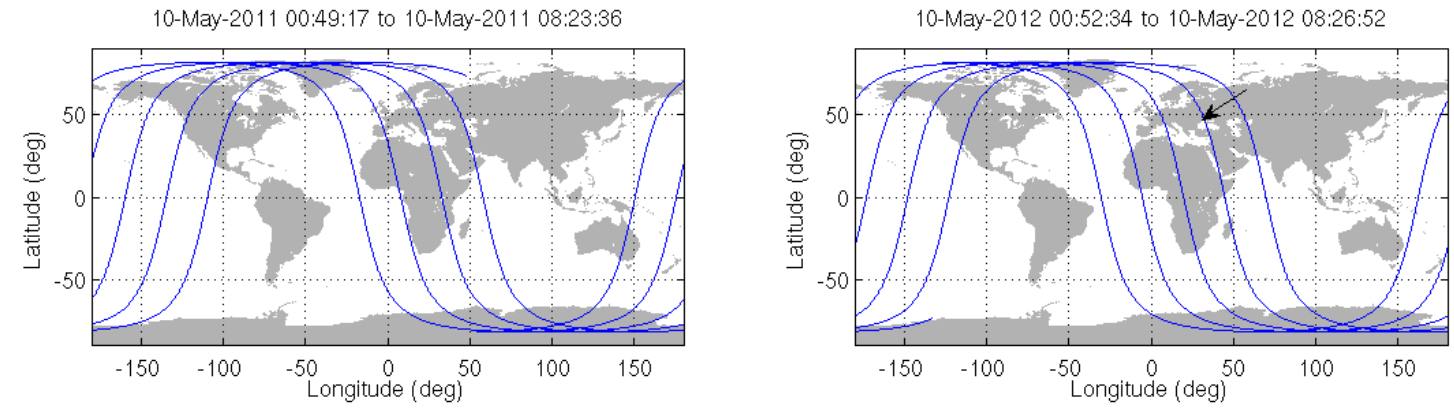

Fig. 5. Orbits used for the Faraday rotation results shown in figure 4.

\section{Conclusions}

Faraday rotation is not negligible in L-Band radiometry and particularly in SMOS it is needed for accurate conversion of brightness temperature from antenna to ground coordinates. In order to improve data processing, it is desirable to retrieve Faraday rotation directly from radiometric data at each snapshot and for all the pixels in the field of view. However, this method becomes highly contaminated by spatial bias and thermal noise, so improved strategies of averaging and data processing are required. 
- To reduce the spatial bias, Faraday rotation has been retrieved as a spatial average within a circular area in the alias-free field of view. A circle of radius $r=0.3$ has been used with good results.

- To reduce the thermal noise, a running average in time has been applied to the Faraday rotation derived at snapshot basis. A triangular filter of width 100 seconds provides sufficient filtering.

With this strategy, the retrieved Faraday rotation tracks well with the theoretical values computed using available ionospheric and geomagnetic field data. On the other hand, the time and space filtering introduced corresponds to a fairly large spatial averaging. As a consequence, the variation of Faraday rotation at a finer scale is lost.

The method works independently over ocean, land or ice, although some ripples are present in land/ice-sea transitions and particularly in zones with high geographical variability the retrievals are worse.

Full-polarimetric SMOS measurement mode (pol-switching technique [4]) and full-polarimetric G-matrix image reconstruction have been used in the processing. The overall results provide evidence that the improvements made to the SMOS processor with regard to the third Stokes parameter [5] probably fixed that problem.

\section{REFERENCES}

[1] H. Barré, B. Duesmann, and Y. Kerr, "SMOS: The mission and the system," IEEE Transactions on Geoscience and Remote Sensing, vol. 46, no. 3, pp. 587-593, March 2008.

[2] K. McMullan, M. Brown, M. Martín-Neira, W. Rits, S. Ekholm, J. Marti, and J. Lemanzyk, "SMOS: The payload,” IEEE Transactions on Geoscience and Remote Sensing, vol. 46, no. 3, pp. 594-605, March 2008.

[3] D. M. Le Vine and S. Abraham, "The effect of the ionosphere on remote sensing of sea surface salinity from space: Absorption and emission at L band," IEEE Transactions on Geoscience and Remote Sensing, vol. 40, no. 4, pp. 771-782, April 2002.

[4] M. Martín-Neira, S. Ribó, and A. J. Martín-Polegre, "Polarimetric mode of MIRAS," IEEE Transactions on Geoscience and Remote Sensing, vol. 40, no. 8, pp. 1755 -1768, August 2002.

[5] L. Wu, F. Torres, I. Corbella, N. Duffo, I. Durán, M. Vall-llossera, A. Camps, S. Delwart, and M. Martín-Neira, "Radiometric performance of SMOS full polarimetric imaging," IEEE Geoscience and Remote Sensing Letters, vol. 10, no. 6, pp. 14541458, November 2013.

[6] S. H. Yueh, "Estimates of faraday rotation with passive microwave polarimetry for microwave remote sensing of earth surfaces," IEEE Transactions on Geoscience and Remote Sensing, vol. 38, no. 5, pp. 2434-2438, September 2000. 
[7] International Association of Geomagnetism and Aeronomy, Working Group V-MOD, "International geomagnetic reference field: the eleventh generation,” Geophysical Journal International, vol. 183, no. 3, pp. 1216-1230, December 2010. [Online]. Available: http://onlinelibrary.wiley.com/doi/10.1111/j.1365-246X.2010.04804.x/full

[8] I. Corbella, F. Torres, L. Wu, N. Duffo, I. Duran, and M. Martín-Neira, "Spatial biases analysis and mitigation methods in SMOS images," in International Geoscience and Remote Sensing Symposium, IGARSS 2013. Melbourne, Australia: IEEE, 21-26 July 2013, pp. 3145-3418.

[9] I. Corbella, F. Torres, N. Duffo, V. González-Gambau, M. Pablos, I. Duran, and M. Martín-Neira, "MIRAS calibration and performance. results from the SMOS in-orbit commissioning phase," IEEE Transactions on Geoscience and Remote Sensing, vol. 49, no. 9, pp. 3147 - 3155, September 2011.

[10] D. M. Le Vine, S. Abraham, C. Utku, and E. P. Dinnat, "Aquarius third stokes parameter measurements: Initial results," IEEE Geoscience and Remote Sensing Letters, vol. 10, no. 3, pp. 520-524, May 2013. 